\title{
Advanced Oxidation Protein Products and the CLOCK 3111T/C Single Nucleotide Polymorphism in Menopausal Women with Insomnia
}

\author{
Natalya V. Semenova, PhD, ScD*; Irina M. Madaeva, PhD, ScD; Anastasiya S. Brichagina; \\ Olga A. Nikitina, PhD; Sergey I. Kolesnikov, PhD, ScD, Member of the RAS; \\ Lyubov I. Kolesnikova, $\mathrm{PhD}, \mathrm{ScD}$, Member of the RAS \\ Scientific Centre for Family Health and Human Reproduction Problems \\ Irkutsk, the Russian Federation
}

\begin{abstract}
Background: The aim of this study was to assess the dependence of the advanced oxidation protein products (AOPPs) levels on the genotype of the CLOCK 3111T/C SNP in Caucasian menopausal women with and without insomnia.

Methods and Results: The study involved 105 Caucasian menopausal women volunteers aged between 45 and 60 years. The Pittsburgh Sleep Quality Index, PSQI, Insomnia Severity Index (ISI), and Epworth Sleepiness Scale (ESS) were employed. The study of the CLOCK 3111T/C SNP (rs1801260) was performed by PCR. The blood level of AOPPs was detected by IEMA. Based on the results of a clinical-anamnestic examination, the women were divided into two groups: the main (with insomnia) and the control (without insomnia). Given the small number of women carrying the CC genotype of the CLOCK 3111T/C SNP, the CC carriers and $T C$ carriers were combined into one group as the carriers of the minor $3111 \mathrm{C}$ allele.

There were no statistically significant differences in the AOPP levels between carriers of different genotypes (TT genotype and $\mathrm{TC}+\mathrm{CC}$ genotypes) in controls and patients. A comparative analysis of AOPP levels in the women of the main and control groups showed higher AOPP levels in women with insomnia carrying the TT genotype than in the control of the same genotype $(P=0.013)$.

Conclusion: Insomnia in menopausal women is associated with increased protein oxidation only in carriers of the TT genotype of the CLOCK 3111T/C SNP. (International Journal of Biomedicine. 2020;10(4):352-356.)
\end{abstract}

Key Words: $C L O C K$ 3111T/C SNP • protein oxidation $\bullet$ insomnia $\bullet$ menopause

\section{Abbreviations}

AOPPs, advanced oxidation protein products; IEMA, immunoenzymometric assay; LPO, lipid peroxidation; OS, oxidative stress; ROS, reactive oxygen species; SNP, single nucleotide polymorphism

\section{Introduction}

Many physiological and metabolic processes, including the sleep-wake cycle, are controlled by a circadian system. It is known that this system consists of a central pacemaker, the suprachiasmatic nucleus $(\mathrm{SCN})$, and of peripheral oscillators

*Corresponding author: Natalya $V$. Semenova, PhD, ScD. Scientific Centre for Family Health and Human Reproduction Problems, Irkutsk, the Russian Federation. E-mail: natkor_84@, $\underline{\text { mail.ru }}$ found in almost all cell types in brain and body that resonate with circadian cues originating from the $\mathrm{SCN} .^{(1,2)}$ Disruption of the biological clock function accompanied by the separation of connections between local oscillators in different tissues or the central oscillator, leads to neuroendocrine rhythms and behavior malfunction. Some studies have demonstrated age-related changes in circadian rhythms. ${ }^{(3)}$ Moreover, any circadian system changes increase the risk of developing many pathological conditions, including sleep disorders. ${ }^{(4)}$

To date, it has been shown that the hormone melatonin plays an important role in the circadian mechanism. ${ }^{(5)}$ Along 
with its regulatory role in the sleep-wake cycle, melatonin is one of the antioxidants that has more pronounced antioxidant properties than vitamin $\mathrm{E}$ or glutathione. ${ }^{(6)}$ It is possible that changes in melatonin secretion circadian rhythms in patients with sleep disorders can lead to OS, which is a result of an imbalance between the production of free radicals and the

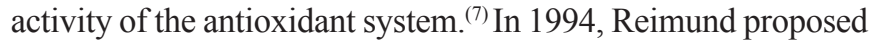
the Free Radical Flux Theory of Sleep. ${ }^{\left({ }^{8}\right)}$ Reimund hypothesized that ROS accumulate in the brain during the wake state, and the lower metabolic rate of sleep provides the brain's antioxidant system the opportunity to catch up, neutralizing neuronal ROS down to baseline levels in preparation for the next day's cycle. The role of OS during sleep was later demonstrated not only by experimental investigations ${ }^{(9-13)}$ but also in human studies, ${ }^{(14,15)}$ including menopausal women. ${ }^{(16)}$ The consequences of LPO product accumulation are changes in the metabolism of proteins, fat, carbohydrates, nucleic acids, water, and electrolyte metabolism, which can cause severe tissue damage and reduction in the adaptive capacity of the organism. ${ }^{(7)}$ It can lead to the development of different cardiovascular, ${ }^{(17)}$ endocrine, ${ }^{(18)}$ and mental diseases, sarcopenia, ${ }^{(20)}$ as well as cancer ${ }^{(21)}$ and other pathologies.

In our earlier study, we found that melatonin secretion circadian rhythms are associated with the CLOCK 3111T/C SNP in Caucasian menopausal women with insomnia. Early morning hours increased the hormone level registered in TT carriers, which allows considering the $3111 \mathrm{~T}$ allele as risky in the formation of melatonin circadian rhythm disturbances in these patients. ${ }^{(22)}$ Moreover, it has been shown that some LPO and antioxidant system parameters in Caucasian menopausal women with insomnia depend on the CLOCK $3111 \mathrm{~T} / \mathrm{C}$ SNP. However, there is no data about the CLOCK $3111 \mathrm{~T} / \mathrm{C}$ SNP and oxidation protein relationships in insomnia.

The aim of this study was to assess the dependence of AOPPs levels on the genotype of the CLOCK 3111T/C SNP in Caucasian menopausal women with and without insomnia.

\section{Materials and Methods}

\section{Subjects}

The study was carried out in compliance with Ethical Principles for Medical Research Involving Human Subjects, Adopted by the 18th WMA General Assembly, Helsinki, Finland, June 1964, and amended by the 64th WMA General Assembly, Fortaleza, Brazil, October 2013. The study was approved by the Ethics Committee of the Scientific Center for Family Health and Human Reproduction Problems. Written informed consent was obtained from each patient.

The study involved 105 Caucasian menopausal women volunteers (the Russian ethnic group) aged between 45 and 60 years, living in Irkutsk city. They were recruited through personal interviews. Once the women had given their written, informed consent to participate in the study, the research program was conducted and included the following methods: clinical-anamnestic, laboratory and statistical.

Inclusion criteria for the perimenopausal group were age of 45-55 years, oligomenorrhea or amenorrhea during 12 months, and ultrasound criteria: (1) endometrial dysfunction (mismatch of structure and thickness corresponding to the first and the second phases of the menstrual cycle); (2) the depletion of ovarian reserve.

Inclusion criteria for postmenopausal group were age of 56-60 years, amenorrhea $\geq 12$ months, follicle-stimulating hormone level $>20 \mathrm{iU} / \mathrm{ml}$, index luteinizing hormone/folliclestimulating hormone $<1$, and ultrasound criteria: (1) thin nonfunctional endometrium, endometrial echo thinner than $5 \mathrm{~mm}$; (2) the lack of ovarian reserve.

Exclusion criteria were exacerbation of chronic diseases, hormone replacement therapy, surgical menopause, the presence of chronic sleep disorders in the history before menopause (insomnia, parasomnia, hypersomnia, obstructive sleep apnea syndrome), the use of hypnotic pills in the previous two weeks, and shift work.

\section{Questionnaires}

The Pittsburgh Sleep Quality Index, ${ }^{(23)}$ PSQI, Insomnia Severity Index (ISI) ${ }^{(24)}$ and Epworth Sleepiness Scale (ESS) ${ }^{(25)}$ were employed. These questionnaires indicate the presence of insomnia according to the criteria of the International Classification of Diseases (ICD-10) and of the Diagnostic and Statistical Manual of Mental Disorders (DSM-IV).

The PSQI questionnaire consists of 19 validated questions for assessment of sleep quality and efficiency. The global PSQI score is calculated from seven components: subjective sleep quality, sleep latency, sleep duration, habitual sleep efficiency, sleep disturbances, use of sleeping medications, and daytime dysfunction. Each question within the component is scored on a 4-point Likert scale of 0-3, with 3 indicating worse outcomes, and the mean was calculated for each component score. The total score ranged from PSQI 0 to 21 points. A global PSQI score 5 indicates a "good sleeper" and $>5$ indicates a "poor sleeper."

The ISI questionnaire consists of 7 items and identifies the severity of sleep continuity disturbance, defined as difficulty initiating sleep and staying asleep, early morning awakenings, and related distress. The answer to each of the questions is scored on a 5-point Likert scale of 0 (none) to 4 (very severe) and a total score ranging from 0 to 28 points. The ISI results 0-7, 8-14, 15-21, 22-28 points were interpreted as the norm, slight sleep disorders, moderate, and severe, respectively.

The ESS consists of 8 items about usual chances of dozing off or falling asleep while engaged in 8 different activities. The answer to each of the questions is scored on a 4-point Likert scale of 0 (not at all likely to fall asleep) to 3 (very likely to fall asleep) and a total score ranging is from 0 to 24 points. The ESS results $0-3,3-9,9-16,16-24$ points were interpreted as norm, insomnia, obstructive sleep apnea syndrome, and narcolepsy, respectively.

\section{Blood collection}

Venous blood was sampled from the cubital vein into two tubes with EDTA tripotassium salt between 8:00 a.m. and 9:00 a.m. after 12-h overnight fasting. Venous blood from the first tube was used for molecular-genetic examination. The sample from second tube was centrifuged for $10 \mathrm{~min}$ at 1.500 $\mathrm{g}$ at $4^{\circ} \mathrm{C}$. Blood plasma was kept frozen at $-40^{\circ} \mathrm{C}$ for up to one month until AOPP determination. 


\section{DNA testing}

Genomic DNA samples were isolated from the peripheral blood leukocytes using the AmpliPrime DNA-Sorb-B reagent kit (NEKSTBIO, Russia). The study of the CLOCK 3111T/C SNP (rs1801260) was performed by PCR on the DTprime amplifier (DNA-Technology, Russia) using reagent sets for genotyping polymorphic markers (TestGen, Russia).

\section{AOPPs determination}

AOPPs blood level (nmol/l) was detected by IEMA using ImmunDiagnostik AG kits (Germany) on a Bio Tek EL $\times 808$ analyzer (USA).

Based on the results of a clinical-anamnestic examination, the women were divided into two groups: the main (with insomnia) and the control (without insomnia). Given the small number of women carrying the CC genotype of the CLOCK 3111T/C SNP, the CC carriers and TC carriers were combined into one group as the carriers of the minor $3111 \mathrm{C}$ allele. The basic characteristics of the groups are demonstrated in Table 1.

Statistical processing was carried out using the STATISTICA Version 10 (StatSoft, USA). The normality of distribution of continuous variables was tested by Shapiro-Wilk test. Mean \pm standard deviation (SD), median (Me), interquartile range (IQR; 25th to 75th percentiles) were calculated. The Mann-Whitney U-Test was used to compare differences between two independent groups. A value of $P<0.05$ was considered significant.

\section{Table 1.}

The basic characteristics of the groups

\begin{tabular}{|c|c|c|c|c|}
\hline \multirow{3}{*}{ Characteristics } & \multicolumn{2}{|c|}{ Control } & \multicolumn{2}{|c|}{ Insomnia } \\
\hline & $\begin{array}{c}3111 \mathrm{~T} / \mathrm{T} \\
(\mathrm{n}=16)\end{array}$ & $\begin{array}{c}3111 \mathrm{~T} / \mathrm{C}+ \\
3111 \mathrm{C} / \mathrm{C} \\
(\mathrm{n}=20)\end{array}$ & $\begin{array}{c}3111 \mathrm{~T} / \mathrm{T} \\
(\mathrm{n}=33)\end{array}$ & $\begin{array}{c}3111 \mathrm{~T} / \mathrm{C}+ \\
3111 \mathrm{C} / \mathrm{C} \\
(\mathrm{n}=36)\end{array}$ \\
\hline & \multicolumn{4}{|c|}{$\mathrm{n}(\%)$} \\
\hline Perimenopause & $7(43.75)$ & $8(40)$ & $15(45.45)$ & $16(44.44)$ \\
\hline Postmenopause & $9(56.25)$ & $12(60)$ & $18(54.55)$ & $20(55.56)$ \\
\hline \multicolumn{5}{|c|}{ Mean \pm SD } \\
\hline Age, yr & $53.75 \pm 5.68$ & $53.35 \pm 5.66$ & $54.85 \pm 4.99$ & $54.75 \pm 5.01$ \\
\hline BMI, $\mathrm{kg} / \mathrm{m}^{2}$ & $27.45 \pm 4.27$ & $26.41 \pm 3.57$ & $28.14 \pm 1.32$ & $27.16 \pm 2.23$ \\
\hline PSQI (points) & $2.32 \pm 0.41$ & $2.11 \pm 0.15$ & $16.27 \pm 2.33^{*}$ & $15.74 \pm 3.04^{\wedge}$ \\
\hline ISI (points) & $3.71 \pm 1.81$ & $4.56 \pm 1.18$ & $24.41 \pm 1.11 *$ & $22.91 \pm 1.17^{\wedge}$ \\
\hline ESS (points) & $1.63 \pm 0.28$ & $1.03 \pm 0.14$ & $7.42 \pm 1.25^{*}$ & $6.65 \pm 1.98^{\wedge}$ \\
\hline
\end{tabular}

$*_{-}<0.05$ between $3111 T /$ T carriers in control and insomnia $\wedge_{-}<0.05$ between $3111 T / C+3111 C / C$ carriers in control and insomnia

\section{Results}

AOPPs levels in menopausal women with insomnia and in the control group, regardless of the CLOCK $3111 \mathrm{~T} / \mathrm{C}$ SNP, are presented in Figure 1. No differences between control and main group were found.

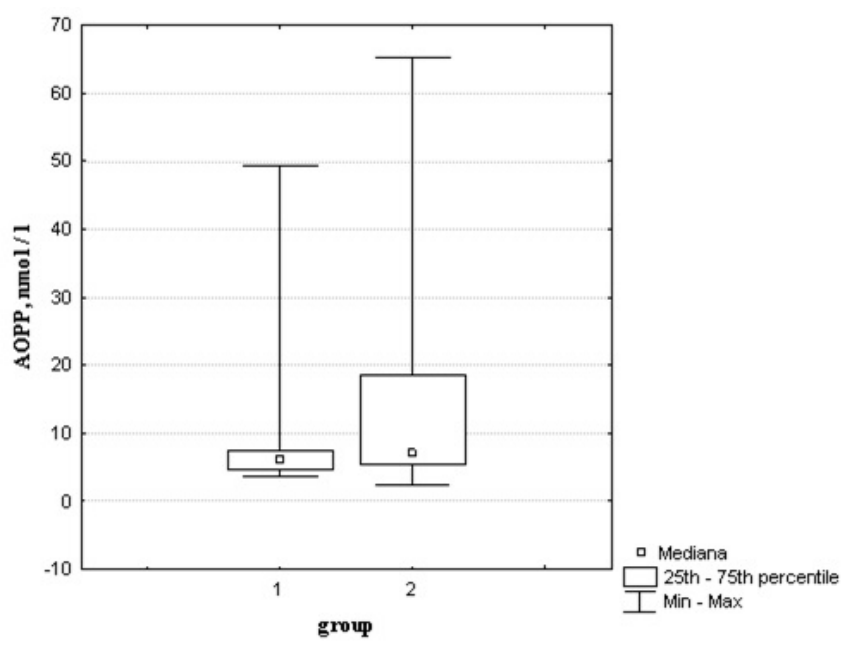

Fig. 1. AOPP levels in control group and women with insomnia

Note: 1 - control; 2 -insomnia

AOPPs levels in menopausal women with different genotypes of the CLOCK $3111 \mathrm{~T} / \mathrm{C}$ SNP are presented in Figure 2. There were no statistically significant differences in the AOPP levels between carriers of different genotypes (TT genotype and $\mathrm{TC}+\mathrm{CC}$ genotypes) in controls and patients. A comparative analysis of AOPP levels in the women of the main and control groups showed higher AOPP levels in women with insomnia carrying the TT genotype than in the control of the same genotype $(P=0.013)$.

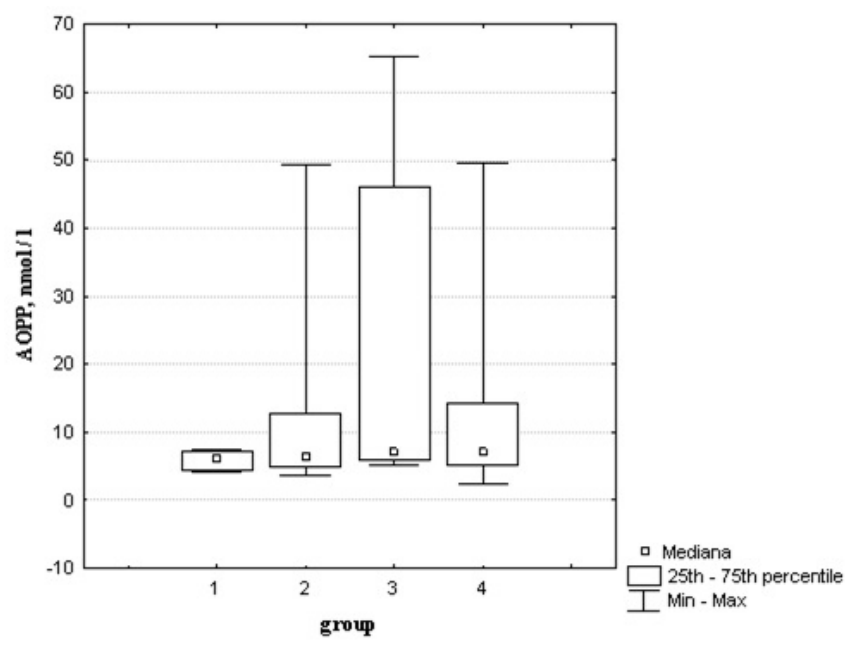

Fig. 2. AOPPs levels in menopausal women with different genotypes of the CLOCK $3111 \mathrm{~T} / \mathrm{C} \mathrm{SNP}$

Note: 1 - control, TT-genotype; 2 - control, TC-, CCgenotypes; 3 - insomnia, TT-genotype; 4 - insomnia, TC-, $\mathrm{CC}$-genotypes

\section{Discussion}

Proteins are one of the main targets of ROS, and their damage associated with the impossibility of repairing most protein damage exerts a significant effect on cell viability. ${ }^{(26)}$ Damaged proteins can cross-link and be involved in the pathogenesis of various diseases. Intensified oxidative modification of 
proteins and increased concentration of AOPPs are confirmed by many experimental investigations in different pathological states, especially those with well-known participation of OS in pathogenesis. ${ }^{(27)}$

Previously ambiguous results about the relationship between OS and insomnia have been shown in some experimental ${ }^{(12,13)}$ and human studies. ${ }^{(14,15)}$ The results of the studies involving menopausal women with insomnia have demonstrated increased levels of TBARS and similar outcomes with control antioxidant system parameters in patients..$^{(16,28)}$ However, further research showed that higher TBARS levels were identified in menopausal women with insomnia who were TT carriers of the CLOCK $3111 \mathrm{~T} / \mathrm{C}$ SNP, while carriers of the minor $C$ allele had only primary LPO products at a high level. ${ }^{(29)}$

The results of our study demonstrated that protein oxidation in menopausal women with insomnia also depends on the CLOCK $3111 \mathrm{~T} / \mathrm{C}$ SNP. These data confirm the development of OS in TT carriers with insomnia. A possible reason for this may be a shift in the melatonin secretion rhythms in these patients. ${ }^{(22)}$ In addition, an increase in AOPP levels combined with a decrease in melatonin levels has been demonstrated in several studies. ${ }^{(30)}$ Another possible reason for the development of OS could be differences in activities of proteasome and lysosomal systems for irreversibly damaged protein utilization and amino acid reuse for continuous protein synthesis in the body. It is known that their proteolytic activity significantly decreases with age. ${ }^{(26)}$

Based on our results, it can be assumed that the 3111C allele is protective for excessive protein oxidation in menopausal women with insomnia. Insomnia in menopausal women is associated with increased protein oxidation only in carriers of the TT genotype of the CLOCK $3111 \mathrm{~T} / \mathrm{C}$ SNP. Preventing OS leading to AOPP accumulation and various pathological conditions is important for menopausal women carrying the TT genotype of the CLOCK $3111 \mathrm{~T} / \mathrm{C}$ SNP.

\section{Competing Interests} interests.

The authors declare that they have no competing

\section{Sources of Funding}

The reported study was funded by the Russian Foundation for Basic Research (Project No 20-415-380001) and the Government of the Irkutsk Region.

This work was performed with the use of equipment of the collective research center "Centre for the development of progressive personalized health technologies" SC FHHRP, Irkutsk.

\section{References}

1. Eckel-Mahan K, Sassone-Corsi P. Metabolism and the circadian clock converge. Physiol Rev. 2013 Jan;93(1):10735. doi: 10.1152/physrev.00016.2012.

2. Lowrey PL, Takahashi JS. Genetics of the mammalian circadian system: photic entrainment, circadian pacemaker mechanisms, and posttranslational regulation. Annu Rev Genet 34: 533-562, 2000.

3. Wennberg AM, Canham SL, Smith MT, Spira AP. Optimizing sleep in older adults: treating insomnia. Maturitas. 2013 Nov;76(3):247-52. doi: 10.1016/j.maturitas.2013.05.007. 4. Feng D, Lazar MA. Clocks, metabolism, and the epigenome. Mol Cell. 2012 Jul 27;47(2):158-67. doi: 10.1016/j.molcel.2012.06.026.

5. Xie Z, Chen F, Li WA, Geng X, Li C, Meng X, et al. A review of sleep disorders and melatonin. Neurol Res. 2017 Jun;39(6):559-565. doi: 10.1080/01616412.2017.1315864.

6. García JJ, López-Pingarrón L, Almeida-Souza P, Tres A, Escudero P, García-Gil FA, Tan DX, Reiter RJ, Ramírez JM, Bernal-Pérez M. Protective effects of melatonin in reducing oxidative stress and in preserving the fluidity of biological membranes: a review. J Pineal Res. 2014 Apr;56(3):225-37. doi: 10.1111/jpi.12128.

7. Sies H. Oxidative stress: a concept in redox biology and medicine. Redox Biol. 2015;4:180-3. doi: 10.1016/j. redox.2015.01.002.

8. Reimund E. The free radical flux theory of sleep. Med Hypotheses. 1994 Oct;43(4):231-3. doi: 10.1016/03069877(94)90071-x.

9. Gopalakrishnan A, Ji LL, Cirelli C. Sleep deprivation and cellular responses to oxidative stress. Sleep. $2004 \mathrm{Feb}$ 1;27(1):27-35. doi: 10.1093/sleep/27.1.27.

10. Mathangi DC, Shyamala R, Subhashini AS. Effect of REM sleep deprivation on the antioxidant status in the brain of Wistar rats. Ann Neurosci. 2012 Oct;19(4):161-4. doi: 10.5214/ans.0972.7531.190405.

11. Ramanathan L, Hu S, Frautschy SA, Siegel JM. Shortterm total sleep deprivation in the rat increases antioxidant responses in multiple brain regions without impairing spontaneous alternation behavior. Behav Brain Res. 2010 Mar 5;207(2):305-9. doi: 10.1016/j.bbr.2009.10.014.

12. Süer C, Dolu N, Artis AS, Sahin L, Yilmaz A, Cetin A. The effects of long-term sleep deprivation on the long-term potentiation in the dentate gyrus and brain oxidation status in rats. Neurosci Res. 2011 May;70(1):71-7. doi: 10.1016/j. neures.2011.01.008.

13. Thamaraiselvi K, Mathangi DC, Subhashini AS. Effect of increase in duration of REM sleep deprivation on lipid peroxidation. Int J Biol Med Res. 2012;3(2):1754-1759.

14. Gulec M, Ozkol H, Selvi Y, Tuluce Y, Aydin A, Besiroglu L, Ozdemir PG. Oxidative stress in patients with primary insomnia. Prog Neuropsychopharmacol Biol Psychiatry. 2012 Jun 1;37(2):247-51. doi: 10.1016/j.pnpbp.2012.02.011.

15. Liang B, Li YH, Kong H. Serum paraoxonase, arylesterase activities and oxidative status in patients with insomnia. Eur Rev Med Pharmacol Sci. 2013 Sep;17(18):2517-22.

16. Hachul de Campos H, Brandão LC, D'Almeida V, Grego BH, Bittencourt LR, Tufik S, Baracat EC. Sleep disturbances, oxidative stress and cardiovascular risk parameters in postmenopausal women complaining of insomnia. Climacteric. 2006 Aug;9(4):312-9. doi: 10.1080/13697130600871947.

17. Sinha N, Dabla PK. Oxidative stress and antioxidants in hypertension-a current review. Curr Hypertens Rev. 2015;11(2):132-42. doi: 10.2174/1573402111666150529130 922.

18. Kolesnikova LI, Darenskaya MA, Semenova NV, Grebenkina LA, Suturina LV, Dolgikh MI, Gnusina SV. Lipid peroxidation and antioxidant protection in girls with type 1 
diabetes mellitus during reproductive system development. Medicina (Kaunas, Lithuania). 2015. 51(2):107-111.

19. Morris G, Stubbs B, Köhler CA, Walder K, Slyepchenko A, Berk M, Carvalho AF. The putative role of oxidative stress and inflammation in the pathophysiology of sleep dysfunction across neuropsychiatric disorders: Focus on chronic fatigue syndrome, bipolar disorder and multiple sclerosis. Sleep Med Rev. 2018. S1087-0792(17):30152-30161.

20. Bellanti F, Romano AD, Lo Buglio A, Castriotta V, Guglielmi G, Greco A, Serviddio G, Vendemiale G. Oxidative stress is increased in sarcopenia and associated with cardiovascular disease risk in sarcopenic obesity. Maturitas. 2018 Mar;109:6-12. doi: 10.1016/j.maturitas.2017.12.002.

21. Kudryavtseva AV, Krasnov GS, Dmitriev AA, Alekseev BY, Kardymon OL, Sadritdinova AF, Fedorova MS, Pokrovsky AV, Melnikova NV, Kaprin AD, Moskalev AA, Snezhkina AV. Mitochondrial dysfunction and oxidative stress in aging and cancer. Oncotarget. 2016 Jul 19;7(29):44879-44905. doi: 10.18632/oncotarget.9821.

22. Semenova NV, Madaeva IM, Bairova TA, Zhambalova RM, Sholokhov LF, Kolesnikova LI. Association of the melatonin circadian rhythms with clock 3111T/C gene polymorphism in Caucasian and Asian menopausal women with insomnia. Chronobiol Int. 2018 Aug;35(8):1066-1076. doi: 10.1080/07420528.2018.1456447.

23. Buysse DJ, Reynolds CF 3rd, Monk TH, Berman SR, Kupfer DJ. The Pittsburgh Sleep Quality Index: a new instrument for psychiatric practice and research. Psychiatry Res. 1989 May;28(2):193-213. doi: 10.1016/0165-1781(89)90047-4.
24. Savard J, Simard S, Morin C. Insomnia. In A. Nikcevic, A. Kuczmierczyk, M. Bruch, editors. Formulation and treatment in clinical health psychology. London: Routledge; 2006.

25. Johns MW. A new method for measuring daytime sleepiness: the Epworth sleepiness scale. Sleep. 1991 Dec;14(6):540-5. doi: 10.1093/sleep/14.6.540.

26. Höhn A, König J, Grune T. Protein oxidation in aging and the removal of oxidized proteins. J Proteomics. 2013 Oct 30;92:132-59. doi: 10.1016/j.jprot.2013.01.004.

27. Piwowar A. [Biochemical and clinical aspects of advanced oxidation protein products in kidney diseases and metabolic disturbances]. Postepy Hig Med Dosw (Online). 2014 Feb 6;68:179-90. doi: 10.5604/17322693.1088754. [ Article in Polish\}.

28. Semenova NV, Madaeva IM, Kolesnikov SI, Solodova EI, Kolesnikova LI. Insomnia in peri- and postmenopausal women: plasma lipids, lipid peroxidation and some antioxidant system parameters. Neuropsychiatry. 2018;8(5):1452-1460. doi: 10.4172/Neuropsychiatry.1000477

29. Semenova N, Madaeva I, Bairova T, Kolesnikov S, Kolesnikova L. Lipid peroxidation depends on the clock $3111 \mathrm{~T} / \mathrm{C}$ gene polymorphism in menopausal women with Insomnia. Chronobiol Int. 2019 Oct;36(10):1399-1408. doi: 10.1080/07420528.2019.1647436.

30. Kratz EM, Piwowar A. Melatonin, advanced oxidation protein products and total antioxidant capacity as seminal parameters of prooxidant-antioxidant balance and their connection with expression of metalloproteinases in context of male fertility. J Physiol Pharmacol. 2017 Oct;68(5):659-668. 